\title{
Intelligent Tutoring Systems: Why Teachers Abandoned a Technology Aimed at Automating Teaching Processes
}

\author{
Marie Utterberg Modén \\ University of Gothenburg \\ marie.utterberg@ait.gu.se
}

\author{
Martin Tallvid \\ University of Gothenburg \\ martin.tallvid@ait.gu.se
}

\author{
Johan Lundin \\ University of Gothenburg \\ johan.lundin@ait.gu.se
}

\author{
Berner Lindström \\ University of Gothenburg \\ berner.lindstrom@ait.gu.se
}

\begin{abstract}
Expectations that technology will improve and streamline education are high. However, technology often introduces new problems. This study aims to explore the challenges mathematics teachers encounter when they implement a digital mathematics textbook with an integrated intelligent tutoring system. A formative intervention was conducted in a two-year project with 16 secondary school teachers. The method was based on activity theory and required the teachers to collaborate with researchers in analyzing their work activity when the new teaching tool was introduced. In this paper, we show that an intelligent tutoring system created systemic contradictions for the teachers. Those contradictions involved predictability, division of labor, individual versus collective learning, accountability, and expectations versus experience. The teachers all tried to resolve the contradictions, but eventually felt compelled to abandon the intelligent tutoring system. The findings contribute to a better understanding of teachers' responses to a technology aimed at automating teaching processes.
\end{abstract}

\section{Introduction}

In education, the number of devices now used in classrooms indicates that the implementation of technology has been successful. Expectations for improvements and transformations have been high; however, these goals have not all been fulfilled [1]. Instead, technology has often introduced new problems or altered the nature of existing problems $[2,3]$. This has sometimes led to teachers being reluctant to use technology in the classroom $[4,5]$.

The purpose of this paper is to explore the changing conditions that arise when teachers use a digital mathematics textbook with an integrated intelligent tutoring system (ITS). Ideally, teachers guide students through subject content in steps that are appropriate to each student. If they teach out of sequence or move on too fast, it is more difficult for the students to progress. Thus, ITSs are designed to present each student with tasks and feedback that are just beyond their existing knowledge. Furthermore, the ITS must be integrated into the existing classroom culture. Since the availability and implementation of ITS has steadily increased, there is a need to understand what effects they have on the teachers' designs for teaching and learning activities [6].

ITSs adapt the content provided to each student, adjusting to the student's current knowledge of a specific domain [6]. The expectation is that the artificial intelligence (AI) applications used in ITSs can support teaching and learning through machine learning and personalized learning. Machine learning is based on algorithms that enable a digital system to automatically learn, often by using training data, to make decisions or predictions [9]. Personalized learning is believed to make learning more efficient and relevant to a student's needs when individual feedback and assessment are used to tailor the instructions [10]. It may seem reasonable to assume that recent development in AI will have an impact on learning and teaching practices. Large amounts of data and increased processing power forecast widespread use [7], while policy documents discuss how AI can influence education [e.g., 8].

Understanding technology mediated change is challenging. The rapid development of technology and the short life cycles of technology in the workplace create the need to consider not just usability and optimization but the whole idea and structure of the work activity [13]. Activity theory provides theoretical tools for analyzing complex socio-technical systems and activities [11]. Contradiction is a core concept in activity theory that we use to understand teachers' use of an ITS in the classroom. Contradictions develop in the teachers' activities when systemic tensions occur within or between the use of cultural historical tools, established norms, and the division of labor. 
Contradictions are manifested in their talk and actions, exposing the disturbance. They provide insights into possible movements and, in so doing, have an important effect on organizational change.

A formative intervention was conducted to enable teachers and researchers to collectively analyze work activity. We used the method Change Laboratory [13] for conducting a formative intervention. This method is particularly useful when studying developmental contradictions as a source of organizational change [12]. In a series of meetings, teachers and researchers together analyzed the work activity, identified the contradictions, and tried to resolve these in order to integrate an ITS.

This paper aims at investigating change conditions for mathematics teachers when they introduce and use an ITS. A Change Laboratory was conducted and was the activity of analysis. Following research question was formulated to guide the work: What contradictions are manifested when mathematics teachers integrate an intelligent tutoring system in their teaching?

\section{Intelligent tutoring systems}

ITSs provide automated adaptivity to students. In this section, we will briefly describe their functionality, how they affect students' performance, and the educational concerns identified in the literature.

\subsection{Functionality}

The key role of the ITS is to automate teaching processes and to optimize students' learning. The intention is to address important indicators of learning and to use this information to personalize automated recommendations for each student's unique learning trajectory and hence, to optimize their learning outcomes.

An ITS simulates a personal tutor, who should understand the individual student's current strengths and weaknesses and closely follow their learning progress [15]. ITSs always include interactivity, adaptivity, and feedback [19]. The system adapts to the student's actions and responds accordingly. It provides information that is determined by the student's knowledge, behavior, and characteristics. The system also gives instant feedback on the student's performance and how it could be improved. An ITS often, but not always, includes choices to encourage self-regulated learning, nonlinear access to learning activities, linked representations to address different conceptual perspectives, and open-ended learner input [16].

In the early 1980s, ITSs were rule-based expert systems within specific domains. Algorithms were based on simple learning principles. The student had to follow the rules created by the expert to understand the subject matter [17]. Emerging ITSs are created to understand learner behavior in terms of statistical inference (conditional probability). Statistics are continuously relying on the student's trajectory and performance and are integrated with machine learning and data mining techniques.

A typical architecture for an ITS consists of three connected models: the domain model, the learner model, and the tutor model $[18,6]$. The domain model acts as an expert in the subject to be learned and what should be taught. It covers the presentation of content knowledge to the student and evaluates student performance. In doing this, the model includes skills, concepts, interrelations, representations, and correct solution strategies. The learner model is a source of comprehensive information about the student. Thus, it captures student features, prior knowledge, and observed behaviors. It represents the student dynamically at a fine-grained level with input taken from the student user data. The tutor model covers pedagogical knowledge about how to teach. It communicates with the domain model and the learner model in deciding how to interact with the student and how to guide the student through an appropriate learning path.

\subsection{Usefulness}

Meta-analyses report optimistic results for ITSs. These studies often compare how outcomes for students in a computer tutoring environment differ from those in a control group with human tutoring. VanLehn [19] shows that an ITS can be almost as effective as a teacher. Kulik and Fletcher [20] find that these systems can outperform conventional teaching. Ma et al. [21] find that ITSs are more effective in terms of students' learning in comparison with large-group instruction led by a teacher and with students working individually with textbooks. Studies on ITSs targeting mathematics have found that struggling students need less teacher assistance [22]. Students using an intelligent tutoring system in algebra and geometry outperformed students using a textbook [23].

However, studies also report mixed results. When ITSs are compared to small group or individual human tutoring, no greater student achievements are found [21]. In mathematics learning, no differences 
were reported between ITSs and regular teaching. However, the effectiveness was greater when the systems were used for just one semester than for a whole school year [24, 25]. A suggestion is that the new tool initially increases engagement and motivation, but that this decreases over time. The effectiveness of ITSs seems to be greater for students in general than for low achieving students [25]. No differences were found among educational levels in mathematics [24], but when the analyses included several subject domains, the ITSs showed better effects on student results in the middle school than in the high school [21].

\subsection{Educational concerns}

ITSs aim to adapt to individual students at a finegrained level, using intelligent algorithms to externalize complex principles of learning [16]. There are examples of what intelligent tutoring systems can do, but there is a danger that when the systems are used at scale they are not representative of the really sophisticated systems. Too often, a claim of AI in educational software turns out to be a simple form of technology involving no AI at all [26, 7]. Many online digital platforms for adaptive learning depend on basic rule-based systems. As such, they must have access to expert knowledge, and the topic to be taught needs to be appropriate for approaches supported by rule-based systems [27, 6]. Consequently, learning activity will be narrowed to units of content that can be structured logically and measured by "making them, not coincidentally, technology-friendly" [28, p. 77].

It is suggested that schools should respond to the 21 st century skills (such as argumentation, communication, and critical thinking) that are needed for students to participate in society and in future employment [29]. Students should be empowered to actively make choices and to take responsibility for their own learning. However, ITSs often have difficulty in representing these complex competencies [6]. There is a risk that creative students will miss novel ideas when the ITSs are trained with data from the past to predict the right conditions for the students' learning. Furthermore, student empowerment could be limited by the students having to rely on ITSs to deliver the instructions [7].

Additionally, these systems rely on the assumption that students should learn in individual and separate paths toward their own goals rather than toward the interest of all students through engagement in a collective classroom. This means that learning is perceived as individual change in incremental steps rather than as a collaborative process taking place in a learning community [28].

\section{Activity theory}

In activity theory, activity is the foundational analytical concept and the entrance point to an understanding of everyday practices. Activity is the unit of analysis and is understood as a dynamic system bridging the gap between humans and technology [14].

\subsection{The object}

An activity is defined by its object. An object gives meaning to the activity. It is the motivating force that directs the activity, and hence, it opens up possibilities and ideas [11]. As such, the object is not a finite individual goal but rather, an unconscious collective orientation, perceived as a sense-maker [30]. The object of an activity is twofold, existing both as a material entity and as an image. It has an independent existence in the world, and it is an envisionment of the object. In this sense, the object is the true motive and defines the activity [31]. Over time, the object changes and will be understood differently by humans involved in the activity through its multifaceted, evolving, and dialogical features [34]. The object is perceived by humans as something that is able to meet a need. When a certain need is met by the object, a motive emerges [31]. This means that the need is transformed, becomes objectified, and "from that moment on, the object becomes a motive and the need not only stimulates but also directs the subject" [33, p. 60].

\subsection{The activity system}

An activity system consists of interrelated components. A subject undertakes actions in an activity. These actions are mediated by tools directed to the object in order to achieve an outcome. Tool mediation is emphasized in activity theory and is based on ideas from Vygotsky [34], who referred to tools as material artefacts and also as non-material mediators, such as signs and symbols. Engeström [35] expands the activity model to include the social context. Rules are regulations and norms affecting a subject's choices to undertake actions in the community of co-participants sharing the same object. Participants in the community are structured into a vertical and horizontal division of labor, based on 
their different roles and responsibilities. The meaning of these components that constitute an activity is established by their possibilities to facilitate the subject in attaining the motive. However, when interacting within an activity, a subject sometimes has motives that are in opposite directions. When the subject is forced to choose between two desirable alternatives, a conflict of motives arises [30]. A conflict of motives can be manifested in different ways, such as uncertainties, dilemmas, tensions, and contradictions. Sannino and Engeström [36, p. 85] describe a conflict of motives as "a clash experienced by an individual between opposite aspirations or tendencies, which occur in situations involving uncertainty and requiring the courage of deliberate choice".

Activity theory is about movement and change. The activity is constantly in transition and contradictions will arise. Contradictions are described as "anything within the system that opposes the overall motive of the system, the aim or purpose that subjects within the system are individually or collectively striving toward" [14, p. 840]. Contradictions are systematic tensions not directly observed but exposed as disturbances in or between activity systems. Contradictions can lead participants to question the situation, which ultimately causes change and improvements [36].

\subsection{Formative intervention}

The Change Laboratory is a formative intervention approach based on activity theory [13]. As such, work is identified as a system of collective object-oriented activity that practitioners can develop by identifying and resolving contradictions [35]. It is important to expose contradictions in order to understand the role of technology in human activities, such as work, and thereby to improve system design [37].

The result of the intervention is not predetermined, rather, the activity is set in motion by researchers and the outcome is determined by the practitioners. Developmental contradictions are explored in relation to components of the activity system. Practitioners collaboratively grasp and analyze invisible structures to get new perspectives for future development [13]. In this process, the voices of all participants in the activity should be heard in the form of debate and negotiation, as multivoicedness [35]. Given increased agency, participants can take action, and a new form of activity will be elaborated that transforms or creates a new layer for the object [13]. This means that the Change
Laboratory is used to promote change and to generate empirical knowledge.

\section{Method}

This paper is based on an on-going two-year research project on the use of digital tools in mathematics education. It explores the challenges faced by mathematics teachers in terms of the contradictions [11] that they encounter when they implement and use a digital mathematics textbook with an integrated intelligent tutoring system.

\subsection{The digital mathematics textbook}

The digital mathematics textbook is an online platform intended to be used over a longer period, for example throughout courses, and it includes a coherent content guided by the mathematics syllabus. All features are integrated into the platform. The platform includes instructional videoclips, mathematical activities, dynamic geometry, automated feedback, and an assessment system, and it has features that contain AI and adaptivity. The content is structured as a tool kit that can be linked, combined, and taught in varied order. Teachers select and assign appropriate features to adapt the system to each student's need.

In addition, the system is self-adapting to the individual student's level of progression. AI is manifested as automated adaptivity in an intelligent tutoring system, and it is integrated as a separate module. A subtopic, or a complete content, can be selected and assigned by the teacher to the students. An intelligent tutoring system recommends mathematical topics and is supposed to assign appropriate tasks for each student based on previous results. Each task is followed by instant correction. If the student does not know an answer, the system will recommend a solution, and a instructional videoclip will be followed by tasks to ensure understanding. For the system to adjust to a student's current knowledge, the student must answer approximately 100 tasks within each subtopic. Consequently, for the system to get a map of a student's knowledge, some tasks will be found too difficult and some too easy according to the student's prior knowledge. Regarding the logic of automated adaptivity, it is not possible for a teacher to predict the tasks that will be given to each individual student.

\subsection{The change laboratory}


Two change laboratories were set up at secondary schools (students aged 13-16). The two schools were located in different municipalities in Sweden. The participants consisted of eight mathematics teachers at each school. The schools were equipped with a 1:1 student to computer ratio. The teachers had varied experience of using a digital mathematics textbook. All the teachers chose to use the digital mathematics textbook as a complement to their paper textbooks. A previous study [3] informed the preparatory fieldwork, that showed problems and challenges for teachers introducing the digital mathematics textbook.

Change Laboratory is based on the method of double stimulation [39]. In this method, two stimuli are presented to the participants by the researchers, termed first and second stimuli. Researchers' fieldwork, in the form of fieldnotes from observations and transcripts from previous Change Laboratory sessions, were used as mirror data. This mirror data was introduced into the Change Laboratory sessions as the first stimulus to highlight disturbances teachers face in their current activity and to trigger conflicts of motives. A phase of questioning was started, in which the teachers expressed their concerns about using the intelligent tutoring system. The second stimulus, an auxiliary stimulus aimed to match and transform the problem or concern, is constructed by the participants and used to face conflicting motives and to gain control of the situation [36]. In so doing, the researchers introduced the triangular model for an activity system, consisting of interrelated components, as the second stimulus. Teachers and researchers, in collaboration, analyzed the activity and identified the contradictions.

In the next step, the potential for using an intelligent tutoring system was explored. Teachers were encouraged to use the intelligent tutoring system in various ways in the course of their teaching. New models for using the system were jointly planned and tested in the classroom by the teachers in an iterative process. The triangular model for the activity was complemented by new models formulated by the teachers. During a workshop, consisted of groupwork, teachers from the two schools together identified disturbances and shared experiences of their new models.

\subsection{Empirical data and analysis}

The first and second authors have been active participants in six Change Laboratory sessions at each school, in total 24 hours. They have also engaged in the field by undertaking, in total, 24 hours of observation in classrooms and by organizing a joint workshop for the teachers from both schools. Data were collected through video recordings, audio recordings, and fieldnotes.

The Change laboratory sessions in the schools were developed in order to promote integration of an intelligent tutoring system in the classroom activities. In change laboratory, contradictions of systemic nature are revealed and dealt with by the involving teachers and researchers acting as process leaders. Hence, Change Laboratory sessions provide data for a deeper analysis of the nature of these contradictions. This was done by a thematic analysis [38]. The analysis gave a possibility to systematically scrutinize empirical data and categorize expressions from participants that we interpreted and understood as manifestations of contradictions in the activity system.

\section{Results}

A central idea of an intelligent tutoring system is to determine what a student knows and to present tasks that are relevant to what the student is now to learn. Recommended videoclips and task solutions will provide one-on-one instruction to help the student understand a mathematical topic. However, an intelligent tutoring system does not necessarily support teachers in their teaching activity and five contradictions were identified involving: (1) predictability, (2) division of labor, (3) individual versus collective learning, (4) accountability, and (5) expectations versus experiences. The teachers had systematically tried to resolve these contradictions. However, they were difficult to resolve, and this left the teachers feeling that they would not use the intelligent tutoring system.

\subsection{Predictability}

Teachers create lesson plans with learning objectives to direct students and to provide a purpose for the learning. The expected long-term goals set by the curriculum and the short-term objectives created by the teacher are articulated to the students prior to the teaching. In practice, specific and clearly defined objectives are provided by paper textbooks. Teachers follow the book, chapter by chapter, and carry out the instructions based on each chapter's content. A teacher explained: "First, I check what this chapter is about. Then I know what they need to know. And then, I design an instruction based on what I know that they need to know about this. Comparing 
fractions for example [...] I check the tasks in the book and then I know briefly what it is all about." In this regard, both content and tasks are well known to the teacher, who can then prepare the students for what they are to do during a lesson.

However, the teachers were unable to predict the students' learning objectives as determined by the intelligent tutoring system. Consequently, they could not align the objectives with their instructions nor address them with the students. The teachers stressed that objectives must be presented to the students so that they know what to do and why. A teacher commented on this issue: "It is difficult to learn anything without a clear goal. The goal cannot be that you, after two years, will have fundamental knowledge. They don't understand what that means. Instead I tell them: this lesson we will talk about purpose and goals, and then, e.g., you should have learned how to convert fractions." In the teachers' experience, using an intelligent tutoring system became increasingly like the students coping with a seemingly never-ending stream of mathematical tasks. A teacher expressed this as: "Automated adaptivity is like Google, it does not act logically. You can lose yourself there. It becomes less distinct what they really need to learn."

In addition, the teachers were unable to predict the content and tasks that would be presented to the students by the intelligent tutoring system. It was a problem for the teachers that they had no idea what the algorithm would present to each student. The tasks are given to the students without the teacher's control. What the students will be doing is a "black box" to the teachers. As one teacher said: "You assign them tasks that are hidden. I have no idea what kind of tasks they get. I experience a lack of control. I prefer control." Thus, when a math's assessment was approaching, the teachers ceased to use the intelligent tutoring system. It was difficult to use in preparation for tests for which the teacher and students needed to work with specific content and tasks.

As a result, the intelligent tutoring system, expected to adapt to each student's need, often assigned content and tasks that the teacher had not yet addressed. Teachers can direct the intelligent tutoring system to present tasks that belong to a specific subtopic. However, such an area is still too broad, according to the teachers, and the students get tasks that are outside of the learning objectives. A teacher summarized this by saying: "It is easier to keep track of what kind of tasks that are upcoming if you have a book [...] And you know that the book won't contain a bunch of weird stuff that they shouldn't be able to manage yet."

\subsection{Division of labor}

Another contradiction concerns the division of labor. Competition emerged between the roles of the teacher and of the intelligent tutoring system, both of which support students with instructions, tasks, and feedback. This is illustrated in the following discussion:

T1: So, what is an AI? Is it something that replaces me, or is it something that complements me? It feels like we have known it as something that is a complement to us. It is a side kick that enters and helps. But it seems as if the AI wants to take over. When we talk about students' learning, for example length and weight. Sure, the AI says: I will fix that.

T2: No, I don't think it will replace us, I never think it will. More likely, being a complement to us.

T1: Yes, I agree. Maybe not replacing us, but it feels like it is designed for that, at the moment.

The teachers agreed that the intelligent tutoring system should assist them and not take over their roles. The intelligent tutoring system should be an assistant that a teacher can communicate with. The teachers should still have control, make the decisions, and take the actions. However, the teachers experience was that the intelligent tutoring system was designed to take over. This feeling is described by a teacher when all the students in the class were using an intelligent tutoring system: "They put headphones on and started to look at videoinstructions and solved a couple of tasks and so on. I just stood there. What should I do? I felt rather unneeded. So, it was a strange feeling. Being in the room, but no one asked me for help [...] It was a strange feeling. The AI suddenly took over [...] I was just lounging around and they were doing their thing. I want to be the one who has control and gives input."

Indeed, the teachers want, and are expected, to help and motivate the students. However, the teachers should not intervene when the students are using the intelligent tutoring system. The system is designed to independently and continuously evaluate the individual student's responses in guiding their learning. Nevertheless, teachers described how they tried to help their students to complete the tasks selected by the automated adaptivity. However, a tension occurred since the system is designed to be autonomous. To meet the needs of all students, the system assigns different tasks in different areas and the teachers have no idea what tasks the algorithm 
will present to each student. Accordingly, when the teachers help the students during a lesson, the teachers must quickly switch between the different types of math task. But it also takes time to explain things individually to each student. The teachers stressed how difficult it was to give feedback to the students when they were given, as the teachers experienced, randomly selected tasks. Talking about this issue a teacher said: "I found it difficult to give feedback to the students. It is very difficult to give feedback when it is very random. When everyone has different tasks, and everyone is at different levels."

In a discussion, the teachers considered what the future might be like if intelligent tutoring systems took over parts of their work as autonomous systems. "They have their headphones on. They sit with the laptops. And instead of walking around and watching, I sit by my desk watching a big screen. So, I can see every student, what they are doing right now, what task. I see exactly what they are doing." Yet, this perspective challenges current classroom norms.

\subsection{Individual versus collective learning}

A prerequisite of the intelligent tutoring system is that students work on their own as the teaching is adjusted to the individual's knowledge level. The teachers are aware of this functionality and explain: "Independent work is what it is all about, otherwise AI will fail. Otherwise, it adapts for someone else, if the student gets help. So, you have to work on your own with this and progress at your own pace." Task solutions and explanatory video clips are recommended to help each student to move on by themselves. A motive that prompted teachers to use the intelligent tutoring system was the greater emphasis on each student and the facilitation of individualization.

However, intelligent tutoring systems are based on students' individual work, whereas the teachers prefer a cohesive environment in the classroom. Teachers plan and conduct their lessons to provide collaborative learning. They stressed that the communication of mathematical thinking plays an important role in learning mathematics. "It is all about communication, communication, discussion, difficult problems, improve skills together, solve. Continuously. That provides quality." Hence, the teachers organize joint discussions and encourage students to work in pairs and to learn together.

Yet, since intelligent tutoring systems are based on the individual student's needs, it makes it difficult to keep the students in a class focused on collective curriculum goals. Intelligent tutoring systems are also based on students' individual learning trajectories. Sometimes this leads to significant variations in the instructions given at different levels and the topics presented to students in the same class. Hence, it is difficult to conduct collaborative activities in the classroom. Commenting on this, a teacher said: "All the talk about teamwork, and the social interaction, it's not there. The student is quite alone."

\subsection{Accountability}

The teachers feel responsible for the students' learning. Their responsibilities include preparing and conducting lessons and assessing and documenting student progress. Consequently, teachers must make informed decisions and be answerable for the decisions they take. However, if intelligent tutoring systems take over these work tasks, teachers still need to be accountable. That is expressed by a teacher: "In the end, the teacher is the last instance, and so it should be. If the parents come to me and ask, then it's me they ask. They don't ask the AI."

The intelligent tutoring system is intended to assign appropriate tasks to each student for their continued learning. However, the teachers lack insights into the system's analysis and how it makes its predictions. The teachers cannot explain the underlying principles affecting the system's strategies, for example, how a student's misconceptions or knowledge gaps are taken into account, and this is particularly important when the assignments given to students seem to have the opposite of the desired effect. A teacher said: "In fact, we hand over our control. But if you hand it over, you want feedback; 'this happened', in such a way that you can understand. And it doesn't, because it hides everything," Another teacher reasoned that: "It should be possible to get feedback on why the AI makes decisions. Why does the AI make the specific decisions? Why does it bring this student up to this level? Why does it move the student to a lower level? The decisions from the AI should be transparent. What makes the AI think that this student should have more difficult tasks in arithmetic? Why? What is the reason for that?" For now, teachers are unable to validate the system's actions, leading to low confidence in the system and a lack of perceived control. Thus, the teachers need to evaluate what the system has decided that the students should learn, and also what they have learned. "How should we find out what happened? Well, we have to ask the student. Why can't we ask the AI? It should be able to tell us." Hence, the teachers are no longer accountable since they cannot justify the system's actions and decisions. 


\subsection{Expectations versus experiences}

Even though the teachers had no previous experience of using an intelligent tutoring system in their teaching, their expectations were influenced by the increased interest in AI in society. However, the teachers' expectations were not matched by their experiences when using an intelligent tutoring system in the classroom. According to Hrastinski et al. [40], there is no common understanding of what intelligent systems can provide and how they can best be used. Consequently, the teachers talked about "our AI" and "ideal AI". They found it difficult to articulate the object that was constructed from previous experiences [32]. The teachers were aware of the different expectations that exist around AI, as illustrated by a teacher during the workshop: "We addressed the issue of managing expectations. Because when a teacher uses it, then you have to find out what it is that is termed AI. What are students' perceptions? What are principals' perceptions? What do politicians/ responsible authorities have in mind about this, that is now purchased and should do wonders". Given that an intelligent tutoring system could possibly contribute to improvements in teaching activities, the teachers were prepared to explore its functionality. Their motives were the ability of technology to provide individualization, accessible information on the students' performance, and increased student motivation.

\section{Discussion}

The literature reports the benefits of using intelligent tutoring systems in education [19, 20, 21]. But the literature also reports educational concerns $[6,7,26,28]$. Given our results, it seems difficult to integrate an intelligent tutoring system into education. The teachers in the study had been looking forward to using an intelligent tutoring system. They were motivated by the system's ability to facilitate adaptation to each student and to provide information about the students' knowledge. However, when the intelligent system was brought into an activity it became a source of frustration. The teachers tried, collectively, to resolve the contradictions, but they failed. The contradictions changed the teachers' ideas about the outcomes of using an intelligent tutoring system, and this led to a change in their motives and the redirection of activity. The need for teachers to work more effectively could, to some extent, be fulfilled by the use of intelligent tutoring systems; but in general, the teachers abandoned the intelligent tutoring system and used activities in the digital textbook that they could select themselves. The teachers chose the most advantageous features among the available resources in the digital textbook and assigned selected video clips and tasks to the students. After a while, the intelligent tutoring system was mostly used for voluntary work and homework. For some students, the use of an intelligent tutoring system increased motivation.

We will direct attention to two themes relevant for discussion in an attempt to resolve these contradictions. These concern the intelligent tutoring system's ability to respond to the teachers' needs.

The first theme concerns whether the intelligent tutoring system or the teaching activities should be re-designed to align with the other. Self-adaptive systems could enable a transformation in educational practice [7], but a challenge identified by the formative intervention of this study is the need to explore new ways of teaching and learning. In our study, the teachers found it difficult to align the content and instructions delivered by an intelligent tutoring system, based on each student, with their own teaching, based on class-level interest. In line with our results, Murphy [6, p. 6] emphasizes that the "self-paced and mastery-learning features of most ITSs that allow such a system to accommodate a range of different learners and abilities can also pose challenges for teachers who want to integrate ITS instruction as an in-class activity that is part of a broader coherent curriculum." Our study shows that predictability is important to enable teachers to prepare themselves and their students for the classroom activities. What, how, and when the students should learn are fundamental questions teachers are constantly addressing and responding to. Yet, when these questions are delegated to an intelligent tutoring system, it leads to changes in the division of labor. Teachers plan and conduct their instructions to structure the learning, but an intelligent tutoring system in the classroom makes the teachers' role uncertain. The issue of who is responsible and held accountable for preparing, conducting, and documenting students' progress needs to be addressed. Our results highlight the teachers' feeling of being responsible for the students' learning and for creating a supportive environment, despite the fact that the intelligent tutoring system is making the decisions and taking action. In addition, the conditions for these decisions are hidden from the teacher. Regarding the logic of intelligent tutoring systems, it is difficult to design them so that they will explain their decisions and actions [7]. Nevertheless, our study reveals the 
importance of keeping teachers included in the process of decision making.

The second theme concerns whether intelligent tutoring systems should be designed for learning mathematics or for teaching mathematics. The purpose of intelligent tutoring systems is to automate teaching processes and to optimize students' learning [15]. Students can progress at their own pace on their own learning path. Complex patterns of how and when students respond to new information can be identified and analyzed. These understandings can be used in supervised learning to increase the accuracy of machine learning models. However, in addition to supporting the students, these systems could give us new insights into how and when learning actually happens [20]. The ability to discern patterns in data could also be used as augmented intelligence to inform teachers through advanced dashboards when planning and conducting lessons. That is, it could augment teachers in conducting high-quality instruction based on all the students' various abilities. It is necessary to explore whether the best use of this technology in education is as artificial intelligence for the students or as augmented intelligence for the teachers.

In conclusion, there is a challenge in combining two fundamentally different systems in the classroom: a technical system and a social system. The technical system is driven by new technologies to improve efficiency, while the social system, which has had a long and stable tradition, is formed by classroom culture. The systemic contradictions described in this paper go beyond the quality of intelligent tutoring systems. Rather, they expose different levels of adaptivity. An intelligent tutoring system needs to adapt to both the students' learning and the teacher's activity. Further research is needed to explore how intelligent tutoring systems could be designed and integrated into the teachers' instructions.

\section{References}

[1] Cuban L. Oversold and underused. Harvard University Press, USA, 2009.

[2] Selwyn N. Education and technology: key issues and debates. Bloomsbury, New York, 2017.

[3] Utterberg M, Tallvid M, Lundin J, Lindström B. Challenges in mathematics teachers' introduction to a digital textbook: Analyzing Contradictions. Journal of Computers in Mathematics and Science Teaching. 2019;38(4):337-359.
[4] Tallvid M. Understanding teachers' reluctance to the pedagogical use of ICT in the 1:1 classroom. Education and Information Technologies. 2016;21(3):503-19.

[5] Utterberg M, Lundin J. "What is the benefit of that?" Mathematics teachers' motives in discarding digital technology in their teaching. Selected Papers of the IRIS, 2017; 8:76-88.

[6] Murphy RF. Artificial intelligence applications to support K-1 2 teachers and teaching, RAND Corporation. 2019. DOI: https://doi. org/10.7249/PE315.

[7] Tuomi I. The impact of artificial intelligence on learning, teaching, and education: policies for the future, Publications Office of the European Union, 2018.

[8] European Commission. Artificial intelligence: a European $\quad$ perspective, 2018. https://publications.jrc.ec.europa.eu/repository/bitstream/JR C113826/ai-flagship-report-online.pdf

[9] Kühl N, Goutier M, Hirt R, Satzger G. Machine learning in artificial intelligence: towards a common understanding. Hawaii International Conference on System Sciences. 2019, pp. 5236-45.

[10] Pane J, Steiner E, Baird M, Hamilton L. Continued progress: promising evidence on personalized learning. Rand Corporation, 2015.

[11] Engeström Y. Learning by expanding. Cambridge University Press, New York, 2015.

[12] Engeström Y. From design experiments to formative interventions. Theory \& Psychology. 2011;21(5):598-628.

[13] Virkkunen, K, Newnham DS. The Change Laboratory: a tool for collaborative development of work and education. Sense Publishers, Rotterdam, 2013.

[14] Karanasios S. Toward a unified view of technology and activity. Information Technology \& People. 2018; 31(1):134-55.

[15] Herder E, Sosnovsky S, Dimitrova V. Adaptive intelligent learning environments. In Duval E, Sharples M, Sutherland R (Eds.). Technology enhanced learning: research themes. Springer, Switzerland, 2017, pp. 109-14.

[16] Graesser AC, Hu X, Sottilare R. Intelligent tutoring systems. In Fischer F et al. (Eds.). Handbook of the learning sciences. Routledge, New York, 2018, pp. 247-55.

[17] Corbett AT, Koedinger KR, Anderson JR. Intelligent tutoring systems. In Helander M,. Landauer TK, Prabhu P (Eds.). Handbook of human-computer interaction, $2^{\text {nd }} \mathrm{ed}$. Elsevier Science, 1997, pp. 849-74. 
[18] Luckin R, Holmes W. Intelligence unleashed: an argument for AI in education. Pearson Education, London, 2016.

[19] VanLehn K. The relative effectiveness of human tutoring, intelligent tutoring systems, and other tutoring systems. Educational Psychologist. 2011;46(4):197-221.

[20] Kulik JA, Fletcher JD. Effectiveness of intelligent tutoring systems: a meta-analytic review. Review of Educational Research. 2016;86(1):42-78.

[21] Ma W, Adesope O, Nesbit JC, Liu Q. Intelligent tutoring systems and learning outcomes: a meta-analysis. Journal of Educational Psychology. 2014;106(4):901-18.

[22] Craig SD, et al. The impact of a technology-based mathematics after-school program using ALEKS on student's knowledge and behaviors. Computers \& Education. 2013;68:495-504.

[23] Ritter S, Anderson JR, Koedinger KR, Corbett A. Cognitive tutor: applied research in mathematics education. Psychonomic Bulletin \& Review. 2007;14(2):249-55.

[24] Fang Y, Ren Z, Hu X, Graesser AC. A meta-analysis of the effectiveness of ALEKS on learning. Educational Psychology. 2019;39(10):1278-92.

[25] Steenbergen-Hu S, Cooper H. A meta-analysis of the effectiveness of intelligent tutoring systems on $\mathrm{K}-12$ students' mathematical learning., Journal of Educational Psychology. 2013;105(4):970-87.

[26] Luckin R, Edtech: how to spot fake AI for education. 2019 October 22, (Web log post). Retrieved February 11, 2020, from https://www.tes.com/news/edtech-how-spotfake-ai-education

[27] Baker RS. Stupid tutoring systems, intelligent humans. International Journal of Artificial Intelligence in Education, 2016;26(2):600-14.

[28] Boninger F, Molnar A, Saldaña CM. Personalized learning and the digital privatization of curriculum and teaching. National Educational Policy Center, University of Colorado Boulder, 2019.

[29] Organization for Economic Co-operation and Development (OECD), The future of education and skills: Education 2030. 2018. Retrieved: 2020-06-09 from https://www.oecd.org/education/2030/E2030\%20Position\% 20Paper\%20(05.04.2018).pdf

[30] Kaptelinin V. The object of activity: making sense of the sense-maker. Mind, Culture and Activity.

2005;12(1):4-18.
[31] Leontév AN. Activity, consciousness and personality. Englewood Cliffs, NJ: Prentice Hall, 1978.

[32] Foot KA. Pursuing an evolving object: a case study in object formation and identification, Mind, Culture, and Activity, 2002;9(2):132-49.

[33] Kaptelinin V, Nardi BA. Acting with technology: activity theory and interaction design. MIT press, USA, 2006.

[34] Vygotsky LS. Mind in society (Cole M, John-Steiner V, Scribner S, Souberman E, Eds.). MA: Harvard University Press, Cambridge, 1978.

[35] Engeström Y. Studies in expansive learning: learning what is not yet there. Cambridge University Press, New York, 2016.

[36] Sannino A, Engeström Y. Co-generation of societally impactful knowledge in Change Laboratories. Management Learning. 2017;48(1):80-96.

[37] Bødker S. Through the interface: a human activity approach to user interface design. DAIMI Report Series 224, 1991.

[38] Braun V, Clarke V. Using thematic analysis in psychology. Qualitative Research in Psychology. 2006;3(2):77-101.

[39] Sannino A. The principle of double stimulation: a path to volitional action. Learning, Culture and Social Interaction. 2015;6:1-15.

[40] Hrastinski S, Olofsson AD, Arkenback C, Ekström S, Ericsson E, Fransson G, . . . Utterberg M. Critical imaginaries and reflections on artificial intelligence and robots in postdigital K-12 education. Postdigital Science and Education. 2019;1(2):427-45. 